\title{
Modification of Watershed Transformation for Images, Containing Small Objects
}

\author{
Marat Kazanov \\ Computer Science Department, Institute for Systems Analysis Academy of Science of Russia \\ mkazanov@mail.ru
}

\begin{abstract}
We present a new method, which allows successful application of watershed transformation for images containing small objects. The method can be divided into two stages. During the first stage of segmentation a special gradient operator is applied to get the gradient of the image. It gives us a possibility to increase the resolution of the gradient doubly in comparison with the resolution of the original image. We can achieve this by approximation of the first-order derivative not only in image pixels, but also between pixels. During the second stage the author's modification of watershed transformation is applied. This modification enables us make optimal use of the image gradient obtained at the first stage. The method to be introduced was probed on a large amount of images with small details, and showed better results than the traditional watershed algorithm.
\end{abstract}

\section{Introduction}

This paper is devoted to the problem of segmentation of digital images containing small objects. In practice, images often have small or highly detailed objects. To segment such objects is to detect their boundaries precisely. Most of the popular segmentation methods, including watershed transformation [1], do not meet these requirements and could not be successfully applied. However, advantages of watershed transformation are well-known, and so the goal of this work is to develop an approach which would allow successful application of watershed transformation for images with small details.

Watershed transformation may be effectively used for segmentation of grey-scale and color images. Segmentation based on watershed transformation always produces continuous segmentation boundaries. It helps to exclude post-processing, such as edge linking, which is usual for other methods [2]. Another advantage of it is that there exist many fast and computationally efficient algorithms of watershed transformation [3], [4].

Why doesn't watershed transformation give acceptable results for images containing small objects? In practice, watershed transformation is usually applied to the gradient of an image rather than to the image itself. So, at first a gradient operator is applied to an image. It is impossible to detect objects' boundaries correctly when their size is smaller than the size of the gradient operator mask. The reason for that is shift of the object's boundaries [5]. Even the smallest of the traditional gradient masks [6] sized $2 \times 2$ pixels fails to recognize the details of objects sized one or two pixels. At the same time, in practice we often find images with objects of uniform intensity, sized one or two pixels, e.g. small letters and symbols.

We introduce here our modification of the watershed transformation algorithm, allowing successful application of watershed transformation to grey-scale and color images and segmentation of nearly uniform objects with small details up to one pixel.

\section{Special gradient operator}

We can divide our method into two stages. At the first stage we apply a special gradient operator to the image. The second stage represents segmentation of the gradient image by means of a modification of watershed transformation. Let us consider the first stage of our approach, the gradient operator. For simplicity we will first consider the gradient operator in a one-dimensional case, and then extend it to two dimensions.

a)

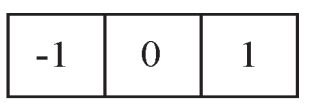

b)

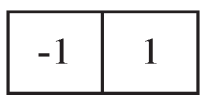

Figure 1. a) 3-pixels first-order derivative mask. b) 2-pixels first-order derivative mask 
Since we want to estimate positions of boundaries very precisely, we will try to estimate with maximum accuracy the value of the first-order derivative. We will estimate it not only at image pixels, but between pixels too. We will use two smallest masks that approximate the first-order derivative of the image. The smaller the mask, the more precisely we can compute the value of the first-order derivative at a point. Figure 1(a) shows the mask we will use for estimation of values of the derivative at each point of the image. This mask is sized 3 pixels. Figure 1(b) shows the mask we will use for the estimation of values of the derivative between image points. This mask has the size of 2 pixels. Thus the value of the derivative at each point of the image will be approximated by the difference between the levels of its left and right neighbors, and the value of the derivative between the points of images will be approximated by the difference between levels of closely situated points.

Let us define our approximation of the first-order derivative operator formally. Let $I(x):=I(i), i=0,1, . . N$ be an original image profile. Let $G(x):=G(j), j=0,1, . .2 N$ be our approximation of the first-order derivative of the image. Resolution of $G(x)$ increases doubly in comparison with the resolution of the original image profile $I(x)$. We define $G(x)$ as:

$$
G(x)=\left\{\begin{array}{l}
\text { even } x: \frac{I\left(\frac{x}{2}+1\right)-I\left(\frac{x}{2}-1\right)}{2} \\
\text { odd } x: I\left(\frac{x+1}{2}\right)-I\left(\frac{x-1}{2}\right)
\end{array}\right.
$$

Figure 2(a) is an example of a one-dimensional image profile, which contains objects sized 5, 2 and 1 pixels. Figure 2(b) and Figure 2(c) show the results of application of the 3-pixels mask and the traditional watershed transformation accordingly. We can see that the boundaries of objects sized 2 and 1 pixels are found incorrectly. Figure 2(d) and Figure 2(e) are the results of the application of the 2-pixels mask and the traditional watershed transformation accordingly. Again, the boundaries of the 1-pixel object are found incorrectly. And finally, Figure 2(f) and Figure 2(g) are the results of applying the combination of two masks and the traditional watershed transformation accordingly. These figures demonstrate that in this case the boundaries of all objects are found correctly. Thus, we introduce now our special gradient operator that consists of the combination of gradient masks sized 3 and 2 pixels.

\section{Modification of watershed transformation}

Let us extend the above-mentioned algorithm to a two-dimensional case. In practice, watershed

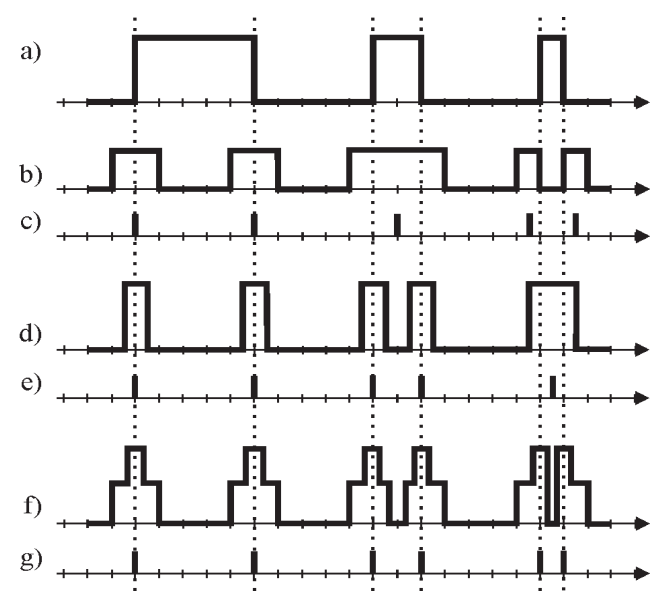

Figure 2. a) Original image profile. b) Result of application of 3-pixels mask to (a). c) Result of segmentation of (b) by means of watershed transformation. d) Result of applicaton of 2-pixels mask to (a). e) Result of segmentation of (d) by means of watershed transformation. f) Result of application of our special operator to (a). g) Result of segmentation of (f) by means of watershed transformation

transformation is usually applied to the gradient image. The intensity at each point is equal to the magnitude of the gradient of the original image. The magnitude of the image gradient $f(x, y)$ at location $(x, y)$ is defined as

$$
\nabla f=\operatorname{mag}(\nabla f)=\left[(\partial f / \partial x)^{2}+(\partial f / \partial y)^{2}\right]^{1 / 2}
$$

As in the one-dimensional case, we will use a 3-pixels mask to compute partial derivatives at the points of the image. To approximate the derivative in $\mathrm{x}$-direction we may use this mask as is shown in Figure 1(a). To approximate the derivative in y-direction we may use this mask, rotating it $90^{\circ}$. But further application of the mask as well as its $90^{\circ}$ rotation is impossible for the approximation of derivatives at positions between pixels.

We will divide the positions between the pixels into three different types according to their location relative to image pixels (Figure 3). Let the original image have resolution of $N \times M$ pixels. Then, after considering the positions between the points of image as pixels, the resolution of the image becomes $(2 N-1) \times(2 M-1)$. We will denote points of images as I-type pixels (Image pixels). Coordinates $(i, j)$ of these pixels can acquire the following values: $i=2 n, j=2 m$, where $n=0,1 . . N ; m=0,1, . . M$. As Htype (Horizontal) pixels we will denote pixels with the following coordinates: $i=2 n+1, j=2 m$, where $n=0,1, . . N$; $m=0,1, . . M$. As V-type (Vertical) pixels we will denote pixels with coordinates: $i=2 n, j=2 m+1$, where $n=0,1, . . N ; m=0,1, . . M$. And finally, as D-type (Diagonal) pixels we will denote pixels with coordinates: $i=2 n+1$, $j=2 m+1$, where $n=0,1, . . N ; m=0,1, . . M$. 
Let us consider finding the gradient of an H-type pixel. Figure 3 shows that we can find an $\mathrm{x}$-directional derivative for an $\mathrm{H}$-type pixel using the 2-pixel mask (see Figure1(b)) the same way as in the one-dimension case. But there are no image points on the vertical line passing through this position, because all the image points lie on both sides of this line. Thus, we must use the mask of a larger size in order to approximate a ydirectional derivative. It is obvious that such a mask with the size larger than the size of the mask for $\mathrm{x}$-directional derivative will give less precise approximation of a $y$ directional derivative.

Now, we estimate in which of the 8 neighboring pixels the accurate directional derivatives can be computed. Figure 3 shows, as was mentioned above, that accurate approximation of the first-order directional derivative for H-type pixels is possible only in the horizontal direction; for V-type pixels accurate approximation of the first-order directional derivative is possible only in vertical direction; for $\mathrm{D}$-type pixels accurate approximation of the first-order directional derivative is possible only in two diagonal directions. Since our original goal is to detect the positions of object's boundaries as accurate as possible, we decided not to compute all the components of the image gradient between pixels. Instead, we decided to use only the precisely detected directional derivatives and to modify the algorithm of watershed transformation accordingly.

In our case however we do not have complete information on all the components of the gradient at each point. Therefore, we would like to introduce the following modification of watershed transformation algorithm for cases, when information about the components of the gradient at some pixels is available in specific directions only.

We could compare our modification of watershed transformation to the rise of water in pipes that slope at different angles. These pipes may meet each other only at certain points. A pipe's altitude at each point equals

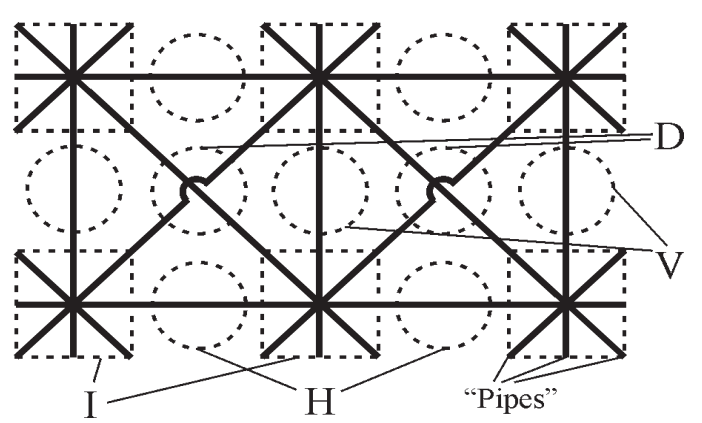

Figure 3. Modified watershed model with pipes

to the value of the directional derivative at this point. The layout of these pipes is presented in Figure 3; it corresponds to the directions, in which derivatives may be estimated accurately.

The main rules of our modification of watershed transformation algorithm are:

1) One should consider a set of pipes of different altitudes instead of the topographical surface used in the traditional watershed transformation algorithm. Altitudes of the pipes are equal to values of directional derivatives.

2) Water in pipes moves only upward;

3) Water in basins used in the traditional watershed transformation algorithm is replaced here by water flows in pipes;

4) Dams are built at the points of possible merging, same as in the traditional watershed transformation algorithm;

5) Merging of flows from different directions is allowed only at the I-type pixels (points of the image), i.e. at pixels, where all gradient components are known; but merging may only be performed after the full flooding of a pixel, i.e. the flooding of the highest pipe at this pixel;

6) It is forbidden to merge different flows at D-type pixels (at pixels, lying on diagonals between the points of the image). a)

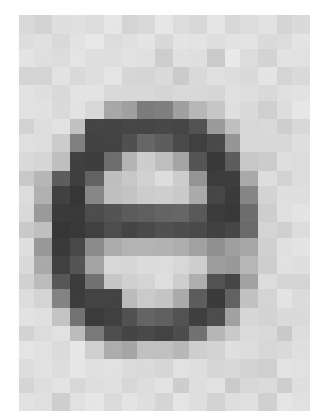

b)

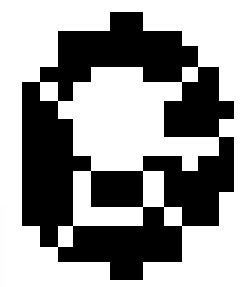

c)

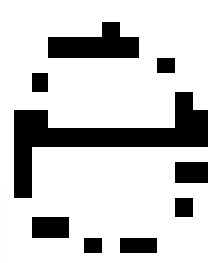

d)

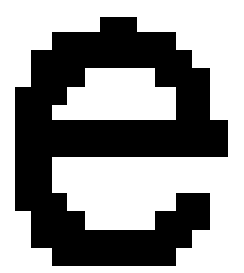

Figure 4. a) Original image. b) Segmentation of (a) obtained by means of watershed transformation with Prewitt mask. c) Segmentation of (a) obtained by means of watershed transformation with Roberts mask. d) Segmentation of (a) obtained by means of our method 
a)

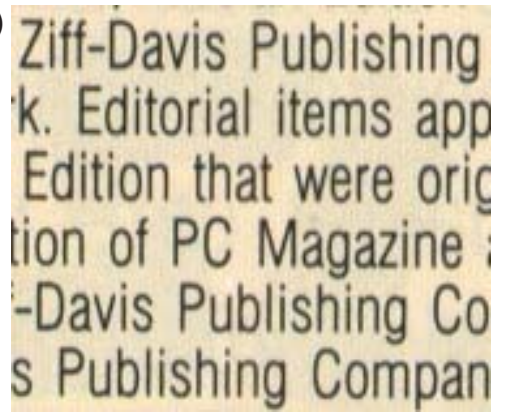

b)

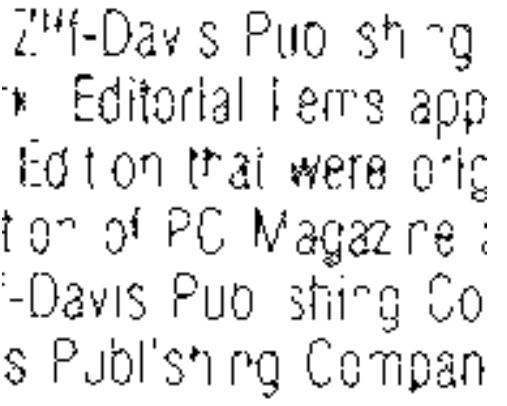

c) Ziff-Davis Publishing k. Editorial items app Edition that were oric tion of PC Magazine : -Davis Publishing $\mathrm{Co}_{0}$ s Publishing Compan

Figure 5. a) Original image with resolution 186x158. b) Segmentation of (a) obtained by means of traditional watershed transformation with Prewitt mask. c) Segmentation of (a) obtained by means of our method

Finally, let us define our algorithm for a twodimensional cases. During the first stage the gradient image is constructed. To implement this a special onedimensional gradient operator consisting of a combination of masks sized 2 and 3 pixels is applied to the original image in the horizontal, the vertical and two diagonal directions. During the second stage, our modification of watershed transformation is applied to the obtained gradient image.

Such modification of watershed transformation allows optimal use of the values of the first-order directional derivatives, as it employs exactly known data only. Simple analysis shows that the result of application of our method to the segmentation of large objects is similar to that performed according to the traditional watershed transformation algorithm. On the contrary, the results of segmentation of smaller objects improve significantly, when our modification of watershed transformation is applied, due to the increase of the gradient image resolution.

\section{Experimental results}

The method to be introduced was probed on a large amount of images with high detail, and yielded better segmentation results at small, nearly uniform objects compared to the results of the traditional watershed transformation algorithm with traditional gradient operator's masks, described in literature [6],[7]. Figure 4(a) shows an enlarged fragment of the original image which contains a small letter "e". Figure 4(b) and Figure 4(c) present the results of segmentation performed by means of the traditional watershed transformation with gradient operator's mask by Prewitt and by Roberts accordingly. Figure 4(d) is the segmentation result obtained by our method. One can notice that the letter "e" was segmented correctly only in the case of application of our method. Figures 5 display the results of segmentation by means of our method compared to the results of the traditional watershed transformation, applied to image containing text.

\section{Conclusion}

We introduce here the method, allowing successful application of the principles of watershed transformation for digital images with small objects. The use of the special gradient operator is underlying the presented algorithm. It allows to approximate the value of the firstorder derivative not only at image points, but also between them. Thus, the resolution of the gradient image increases twice as much compared to the resolution of the original image. To optimize the usage of the obtained gradient image, we propose a modification of watershed transformation. Application of this method allows to detect boundaries of small objects accurately. It is necessary to note that the presented algorithm is noisesensitive. This disadvantage is the subject of our further investigations.

\section{References}

[1] S. Beucher, F. Meyer, The Morphological Approach to Segmentation: The Watershed Transformation, in "Mathematical Morphology in Image Processing", E. R. Dougherty Editor, Marcel Dekker, Inc, New York, pp.433481, 1992.

[2] R.C. Gonzalez, R.E.Woods, Digital Image Processing, Prentice-Hall, Inc, Upper Saddle River, New Jersey, pp. 617626, 2002.

[3] L. Vincent, P. Soille, "Watersheds in Digital Space: An Efficient Algorithm Based on Immersion Simulations", IEEE Trans. Pattern Anal. Mach. Intell 13 (6), pp. 583-598, 1991. [4] A. Bieniek, A. Moga, "An efficient watershed algorithm based on connected components", Pattern Recognition 33, pp. 907-916, 2000.

[5] D. Williams, M. Shah, "Edge Contours Using Multiple Scales", Computer Vision, Graphics, and Image Processing 51, pp. 256-274, 1990.

[6] L.G. Roberts, "Machine Perception of Three-Dimensional Solids", Optical and Electro-Optical Information Processing, Tippet, J.T. (ed.), MIT Press, Cambridge, 1965.

[7] J.M.S. Prewitt, "Object Enhancement and Extraction", Picture Processing and Psychopictorics, Lipkin, B. S., and Rosenfeld, A. (eds.), Academic Press, New York, 1970. 